Meta

Journal des tradlucteurs

Translators' Journal

\title{
La traduction et l'interprétation en Océanie
}

\section{Daniel Gile}

Volume 28, numéro 1, mars 1983

La traduction dans le monde

Translation around the World

URI : https://id.erudit.org/iderudit/002905ar

DOI : https://doi.org/10.7202/002905ar

Aller au sommaire du numéro

Éditeur(s)

Les Presses de l'Université de Montréal

\section{ISSN}

0026-0452 (imprimé)

1492-1421 (numérique)

Découvrir la revue

\section{Citer cet article}

Gile, D. (1983). La traduction et l'interprétation en Océanie. Meta, 28(1), 17-19.

https://doi.org/10.7202/002905ar d'utilisation que vous pouvez consulter en ligne.

https://apropos.erudit.org/fr/usagers/politique-dutilisation/ 


\section{LA TRADUCTION ET \\ L'INTERPRÉTATION EN OCÉANIE}

Daniel Gile

\section{A.PERÇU GÉOLINGUISTIQUE}

L'Océanie se compose de deux pays métropolitains, l'Australie et la Nouvelle-Zélande, exclus de cet exposé, et d'une vingtaine de pays et territoires insulaires situés dans le Pacifique méridional et central. Ces derniers se caractérisent, à quelques exceptions près, par une petite surface des terres émergées, une grande dispersion des archipels et de faibles populations (quelques milliers à cent cinquante mille habitants environ, à l'exception de Fidji, qui en compte un demi-million, et de la Papouasie-Nouvelle-Guinée, qui en compte trois millions et dont la masse terrestre est très importante).

La quasi-totalité des pays mélanésiens (Océanie occidentale) et micronésiens (Océanie septentrionale) comptent au moins quelques dizaines de langues distinctes, intelligibles pour les seules communautés qui les pratiquent. Dans les pays polynésiens, il existe une langue par pays. Les langues de Polynésie orientale sont très proches; les langues de Polynésie occidentale le sont moins.

Les langues vernaculaires sont donc nombreuses et diversifiées dans la région. Elles sont en général employées par de petites communautés linguistiques, souvent isolées.

D'autre part, tous les pays et territoires océaniens, à l'exception du Royaume de Tonga, ont un passé, voire un présent colonial dont ils ont hérité une langue métropolitaine, à savoir le français en Nouvelle-Calédonie, en Polynésie française et à Wallis et Futuna, et l'anglais ailleurs.

\section{LE BILINGUISME OCÉANIEN}

Tous les pays et territoires océaniens sont donc au moins bilingues, avec une ou plusieurs langues vernaculaires et l'anglais ou le français comme langues officielles (l'anglais et le français à Vanuatu).

Dans les pays mélanésiens, on constate un recul des langues vernaculaires dans les centres urbains et un maintien des langues locales, avec une utilisation limitée de la langue coloniale, dans les zones rurales. Dans les pays polynésiens, l'unicité de la langue indigène lui permet apparemment de mieux résister.

Le bilinguisme océanien est compartimenté dans une grande mesure, la langue indigène servant plutôt à ce qui est personnel, affectif et coutumier, et la langue importée au discours intellectuel, technique ou administratif.

L'apprentissage de la langue coloniale est essentiellement scolaire, et la langue vernaculaire s'acquiert en famille. Depuis quelque temps, plusieurs gou- 
vernements déploient des efforts pour donner une nouvelle vie à la langue vernaculaire dans les centres urbains en l'intégrant à l'enseignement scolaire.

\section{LES BESOINS EN MATIËRE DE TRADUCTION ET D'INTERPRÉTATION}

La traduction et l'interprétation sont surtout destinées aux habitants des archipels éloignés qui ne se servent que de leur langue vernaculaire et ne maîtrisent pas la langue métropolitaine. Le besoin s'en fait sentir quand ils ont à accomplir ou à subir des démarches administratives ou judiciaires. C'est dans ces domaines que se concentre une importante partie de l'activité traduisante dans les pays océaniens. Le reste relève des textes et débats parlementaires, respectivement traduits et interprétés en vertu de dispositions constitutionnelles existant dans plusieurs pays de la région.

Enfin, des activités marginales de traduction scolaire et littéraire peuvent être relevées par endroits, mais elles sont nécessairement limitées par la petite taille des populations-cibles et par la faiblesse de leurs moyens économiques.

Quant à la traduction scientifique et technique, elle est très rare, car les seuls utilisateurs de textes de cette nature sont des Océaniens ayant fait des études ou suivi une formation professionnelle dans un pays métropolitain et maitrisant par conséquent la langue coloniale dans laquelle ces documents sont rédigés.

\section{LES TRADUCTEURS ET INTERPRÈTES OCÉANIENS}

Dans la plupart des pays de la région, les seuls traducteurs et interprètes à plein temps sont fonctionnaires parlementaires. Par ailleurs, parmi les effectifs des services judiciaires, on trouve des interprètes et traducteurs «occasionnels». Enfin, la traduction scolaire et littéraire et l'interprétation d' «accompagnement » permettent à de nombreux amateurs de s'exprimer.

Les interprètes-traducteurs-terminologues parlementaires sont fréquemment recrutés parmi d'anciens enseignants, dont la maitrise et l'expérience de la pratique des deux langues sont les seuls éléments de qualification professionnelle. Si la formation sur le tas est la règle, il semblerait que l'université nationale de Papouasie-Nouvelle-Guinée offre des cours d'interprétation. En outre, le «Institute of Pacific Studies» de l'Université du Pacifique Sud organise périodiquement des stages de traduction.

L'interprétation se fait en général en "consécutive», phrase par phrase, les exceptions étant les parlements de Papouasie-Nouvelle-Guinée et des Samoas Occidentales, où les débats parlementaires sont interprétés en simultané.

Les interprètes parlementaires sont aussi chargés de la création de nouveaux mots dans les langues locales pour permettre à celles-ci de véhiculer des concepts essentiellement économiques, administratifs et juridiques correspondant à des termes appartenant à la langue métropolitaine.

Les procédés communément employés sont, comme partout ailleurs, l'emprunt conjugué à la «naturalisation" de la prononciation, la traduction littérale et la création lexicale. Ce travail d'enrichissement est limité aux domaines d'activité des traducteurs parlementaires, ce qui aboutit à un élargissement lexical des langues locales dans ces domaines et à une stagnation ailleurs. 


\section{LA TRADUCTION ET L'INTERPRÉTATION À L'ÉCHELON RÉGIONAL}

La majeure partie des activités de traduction et d'interprétation en Océanie sont donc intra-nationales plutôt qu'internationales, et intéressent au premier chef la communication entre langues vernaculaires et langues coloniales.

À l'échelon régional, les structures sont soit entièrement anglophones, soit bilingues avec le français et l'anglais comme langues officielles.

La Commission du Pacifique Sud, dont le siège se trouve en NouvelleCalédonie, est la seule organisation régionale qui ait une section traduction et interprétation. Cette section se compose de 6 personnes, dont deux traducteurs, trois interprètes-traducteurs et un directeur, recrutés selon des critères identiques à ceux des organisations des Nations-Unies.

L'essentiel de la traduction et de l'interprétation à l'échelon régional est assuré par les traducteurs et interprètes de la CPS, qui organise des conférences techniques, seule ou en collaboration avec d'autres organisations telles que l'OIT, le PNUD ou le SPEC (South Pacific Bureau for Economic Cooperation). La CPS assure également l'interprétation de conférences organisées par d'autres institutions, telles que l'UNESCO ou l'Université du Pacifique Sud.

\section{L'AVENIR DE LA TRADUCTION ET DE L'INTERPRÉTATION EN OCÉANIE}

Le bilinguisme devrait rester une constante géolinguistique de la région pendant de longues années encore, car si la présence pressante de structures administratives et technologiques de type métropolitain donne un poids considérable à la langue coloniale, la dispersion et l'isolement des communautés insulaires assurent la survie des langues vernaculaires qui y sont pratiquées.

D'autre part, la petite taille des populations concernées et leur faible potentiel économique ne permettent pas d'espérer un développement suffisant de la traduction pour réaliser l'intégration de l'ensemble des données importées aux cultures locales.

On peut s'attendre à une lente progression de l'activité de traduction dans certains domaines, mais un bouleversement de la situation nous parait peu probable à court ou moyen terme.

Quant à la mise en place de programmes linguistiques et de traduction dans les différents pays et territoires, nous souhaitons, et c'est là un vœu personnel, qu'y figure toujours en bonne place le souci de conserver, voire de développer ce précieux patrimoine culturel, fragile, menacé et même éteint par endroits, que constituent les langues vernaculaires océaniennes. 